\title{
A Study on Different Compositions of Epoxy Resin Reinforced with Natural Fibers and Glass Fiber
}

\author{
Abrar Rameez Samad, Andrew Athisayaraj E. R, Ashwin Karthik. R, Bharath. R \\ Guided by Mrs. Queen Florence Mary M.E, \\ Assistant Professor \\ Department of Mechanical Engineering \\ Loyola-ICAM College of Engineering and Technology \\ Chennai, India
}

\begin{abstract}
Today the usage of natural fibers as reinforcement in polymer composite material has increased significantly. The natural fibers prove to have wide area of research since it has low density, light weight and better mechanical properties. They are also ecofriendly to certain extend and also available abundantly. In this study testing was done on taking three different combination of composites, the first combination is the epoxy resin reinforced with bamboo fiber, linen fiber, palm fiber and glass fiber. The second combination is the epoxy resin reinforced with Bamboo Fiber, Linen fiber and Glass fiber. In the third combination the matrix is reinforced with Palm Fiber, bamboo fiber and Glass Fiber. The above three combinations are also varied with the weight ratios of the matrix to fiber. All the samples were fabricated using HandLayup Technique and the properties were analyzed. The Impact Strength, Tensile Strength, the Flexural strength, hardness and absorbent test of the composites were performed. The properties of all the specimens were tabulated and compared with the cost analysis of each combinations are performed.
\end{abstract}

Keywords-Epoxy Resin, Palm Fiber, Glass Fiber, Flax, Bamboo Fiber, Mechanical Properties

\section{INTRODUCTION}

Composite material is a macroscopic combination of two or more distinct materials, having a recognizable interface between them. Composites are used not only for their structural properties, but also for electrical, thermal, and environmental applications. Modern composite materials are usually optimized to achieve a particular balance of properties for a given range of applications. Given the vast range of materials that may be considered as composites and the broad range of uses for which composite materials may be designed, it is difficult to agree upon a single, simple, and useful definition. However, as a common practical definition, composite materials may be restricted to emphasize those materials that contain a continuous matrix constituent that binds together and provides form to an array of a stronger, stiffer reinforcement constituent.

The composites are heterogeneous materials, which is an important feature compared for instance to the metal homogeneous plastics. There are many kinds of failure and damage modes in the composite structures. One of them is the interlaminar fracture known as delamination, which is, at the same time one of the most important failure mode. Delamination growth remains a critical failure mode in laminated composite structures. The interlaminar fraction of composite material has been very intensively investigated. The delamination means degradation between adjacent plies of material. The composite materials exhibit superior properties only in the fiber direction; hence the delamination of composite structures results in a significant loss of stiffness and strength. The interlaminar crack growth resistance of long fiber-reinforced composites can significantly increase in the presence of fiber bridging, which induces tractions that act over an extended zone in the wake of the crack tip. The resistance to delamination is one of the most important characteristics of laminate and unidirectional composites. One major drawback of composite materials is delamination-separation of a laminate into layers. One major US Company, Kinetic, failed partly because of delamination failure at the trailing edge. The size of the wind turbine blades, without the proper understanding of the material behavior, is likely to produce failure due to delamination.

Many ways have been found to resist delamination, for example weaving the fibers increases the toughness, but introduces micro-buckling modes, which is detrimental to the compressive strength; toughening the resin suppresses delamination but often decreases the modulus, an inherent trade-off in increasing toughness in the resins. Toughened resins are commonly used in aerospace pre impregnated materials, to resist delamination. However, the cost of using prepreg materials in wind turbine manufacture can be high. Hence, low cost composite materials are sought for building wind turbine blades, such as fiberglass, where delamination has not been studied in detail. There are three fundamental ways delamination can happen: opening mode, shearing or sliding mode, and tearing mode. More often than not, delamination occurs under mixed opening and shearing modes, which is the subject of this study. This study is the extension of researches by Darrin Haugen and Robert More head, who studied delamination of the skinstiffener intersection geometry which is common in 
composite materials structures like wind turbine blades. This work combines, adds to, and revises their earlier work.

\section{MATERIALS USED}

\section{Palm Fiber}

The palm fiber (Borassusflabellifer) is a natural fiber obtained from various portions of palm tree which is found in abundance in the southern parts of India. The palm fibers are composite material designed by nature, the fibers are basically a rigid, crystalline cellulose microfibril- reinforced amorphous lignin and/or with hemicellulose matrix. Most plant fibers are composed of cellulose, hemicellulose, lignin, waxes and some water-soluble compounds. Generally, this fiber contains $60-80 \%$ cellulose, $5-20 \%$ lignin and up to $20 \%$ moisture. The fibers from various parts of the palm tree were extracted either by retting process or mechanical processing or hand processing. The fibers are soaked for two weeks and then it is washed with water and allow it dry completely in natural sunlight to obtain long and dried long uniform fibers. The fibers are extracted from various parts of palm trees are Palm leaf stalk, Palm leaf sheath, Palm fruit, and Palm petiolar respectively. Through research experimental, the palm fruit fiber has good mechanical properties like specific strength, tensile strength, and hardness etc. The palm fiber extracted from palm fruit gives more strength, the tensile behavior of the palm fibers increases with long sized fibers extracted from palm fruit. Glass fiber and carbon fibers have more density than palm fiber, therefore, the specific strength is better for the palm fiber compared with other fibers. Through the various experimental investigations, it has been found, the palm fiber especially the fiber from palm fruit gives more tensile strength and young's modulus compared with other natural fibers. The major compositions present in the palm fiber are cellulose, lignin and pectin. The palm fibers normally have more percentage of cellulose content which enables the fiber with better mechanical strength.

\section{Flax Fiber}

Flax fiber is extracted from the best beneath the surface of the stem of the flax plant. Flax fiber is soft, lustrous, and flexible; bundles of fiber have the appearance of blonde hair, hence the description flaxen hair. Flax fiber is the strongest fiber in cellulosic fibers (plant fibers), two to three times stronger than cotton fiber, therefore it is more resistant to wear and abrasion. Flax fiber is extracted from the skin of the flax stem plant. Flax has the best heat resistance and conductivity. Flax material has the potential of load-bearing constituents in composite materials due to their adjective properties such as high stiffness to weight ratio.

\section{Glass Fiber}

Generally, polymers and carbon fiber have good mechanical properties but in recent evolution, this will be limited to some technological applications. The researchers have found the glass fibers which are extracted from glass having finely sized in the diametral range of 10-25-micron levels will be used in recent the development of composite materials. The glass fibers comparatively have more strength and rigidity and also the cost of glass fiber is low. Therefore, in the recent development in NFC's these glass fibers are majorly used as a reinforcement material to manufacture lightweight and relatively strong composite materials. Lightweight fiberreinforced polymer (FRP) composite material called glass reinforced plastic (GRP), also popularly known as "fiberglass".

\section{Bamboo Fiber}

Traditionally, bamboo has been used in various living facilities and tools, which owes to its high strength and weight. This property is due to the longitudinal alignment of fibers. Bamboo has $60 \%$ cellulose with high content of lignin, and its microfibrillar angle is $2-10$ degree. In practice, it is mandatory to fabricate the bamboo-based composites in addition to the extraction of bamboo fibers in a controlled. Its structural variation, mechanical properties, extraction of fibers, chemical modification and thermal properties had made it versatile for the use in composite. Despite its finer mechanical properties, bamboo fibers are brittle when compared with other natural fibers, which is due to the extra lignin content.

\section{MANUFACTURING METHOD USED}

\section{Hand-layup Process}

The composite materials used for the present investigation is fabricated by hand layup process. In this work, Initially, the fiber mats of uniform thickness were prepared with flax and palm fibers. The composite laminate consists of multilayers. The mats were impregnated with epoxy resin then; the fibers are dried under the hot sun to remove their moisture for more than 24 hours. Now, the fiber layers are washed in the acetone thinner before they are used for fabrication. Acetone removes the impurities and makes them ready for binding with the resin releasing agent is applied on the top of the table. The Flax fibers are spread on the base plate of the molding box which is placed on the table. It is then completely spread with epoxy resin and hardness mixture is applied on the flax fiber with brush and smoothens with a roller. Then, a layer of GFRP is placed on the resin-hardener mixer above which next layer of flax fiber is spread. Similarly, multiple layers are placed to prepare the composite (3-layer Flax/palm and 4 Layer GFRP). In this review, it is planned to prepare 3 types of composite. Type 1 composite contains all fibers arranged inclined at $45^{\circ}$ to each other in all the 3 layers which resemble inclined orientation. Type 2 composite contains flax-fiber. Type 3 contains all fibers except flax fiber. In each layer, both fibers are tilted at 45 degree in the subsequent layer (45degree orientation). In all the types, the top and bottom most layers is GFRP.

\section{RESULTS}

All the material samples were fabricated and various important tests were conducted on the samples that were fabricated. The various tests conducted include tensile test, hardness test, impact test, flexural strength test and lastly water absorption test. The test results are tabulated in a table and is presented below along with a graph representation. 


\section{DISCUSSION}

A lot of research has been done on natural fiber reinforced polymer composites but research on palm-based polymer composites is very rare. Against this background, the present research work has been undertaken. Palmyra individual fiber is a better choice than other different fiber composites because it shows highest ultimate tensile stress under various tests performed. From table 1 it has been noted that the palm fiber and glass fiber have more strength and increased mechanical properties. This review deals with an objective to explore the potential of palm fiber, bamboo fiber and flax fiber composites reinforced with glass fiber for better mechanical properties mainly focused on strength and hardness of the composites. The experimental investigation on the composite lamina samples for various mechanical properties test in the future work. In future, there are tremendous investments on natural fibers, to bring a great impact on manufacturing sectors.

\section{CONCLUSION}

In recent day's lots of researchers progressed to improve the mechanical characteristics of natural fiber composites. The improvement is been made by fiber selection, extraction and treatment given to the fibers as well as processing of composite by proper selection of coatings over it. Comparing natural fiber and glass fiber reinforced composites found that natural fibers were superior in industrial application. Moreover, due to the usage of natural fibers in different engineering application and construction industries, it provides a way for economic development in rural areas. This paper reviewed the research that has mainly focused on improving strength, stiffness and impact strength. The major objective of the review paper is increasing the tensile strength of natural fiber composite by coating it with glass fiber. Since glass fiber have the adequate tensile strength and impact strength nature the mechanical properties of natural fiber composite (Palmyra-spout and flax fiber) reinforced with glass fiber is increased. Applications of natural fiber composites have extended dramatically including load bearing and outdoor applications such as automotive exterior underfloor paneling, sports equipment, rooftops and marine structures.

Further, the experimental investigation to be done over the samples and through the results various mechanical properties is concluded. Overall, the growth of natural fiber composite is growing rapidly and shown to be very positive future ahead of their application.

\section{REFERENCES}

[1] K. L. Pickering, M. G. AruanEfendy, T. M. Le, (2016) A review of recent developments in natural fibre composites and their mechanical performance. Part A 83 (2016) 98-112.

[2] Malhotra, N., Sheikh, K. and Rani, S. (2012) A Review on Mechanical Characterization of Natural Fiber Reinforced Polymer Composites. Journal of Engineering Research and Studies, 3, 75-80.

[3] K. P. Ashik, Ramesh S. Sharma. "A Review on Mechanical Properties of Natural Fibre Reinforced Hybrid Polymer Composites", Journal of Minerals and Materials Characterization and Engineering, 2015, 3, 420-426.

[4] Chandramohan, D. and Marimuthu, K. "A Review on Natural Fibers"(2011) IJRRAS, 8, 194-206.

[5] Dash, D., Samanta, S., Gautam, S. S. and Murlidhar, M. (2013) Mechanical Characterizations of Natural

[6] FiberReinforced Composite Materials. Advanced Materials Manufacturing \& Characterization, 3.

[7] Ramesh, M., Palanikumar, K. and Reddy, K. H. (2013) Mechanical Property Evaluation of Sisal-Jute-Glass Fiber Reinforced Polyester Composites. Composites: Part B, 48, 19.2012.12.04 K. P. Ashik, R. S. Sharma 426

[8] Amit Rai et al Int. Journal of Engineering Research and Applications, Applications and Properties of Fibre Reinforced Concrete ISSN: 2248-9622, Vol. 4, Issue 5(Version 1), May 2014, pp.123-131

[9] Holbery, J., Houston, D., Natural-Fiber-Reinforced Polymer Composites in Automotive Applications, JOM, 2006, 11, 80-86.

\begin{tabular}{|l|c|c|c|}
\hline \multicolumn{1}{|c|}{ Test } & Sample 1 & Sample 2 & Sample 3 \\
\hline Tensile Strength, $(\mathrm{MPa})$ & 4.74 & 35.98 & \\
\hline Sheer Hardness, $(\mathrm{D})$ & 50 & 46 & \\
\hline Impact, $(\mathrm{J})$ & 14 & 3.96 & \\
\hline Flexural Strength, $(\mathrm{MR})$ & 7.25 & 5.30 & 4.6 \\
\hline Absorption, $($ Th\% $)$ & 33.33 & 31.82 & 5.69 \\
\hline
\end{tabular}

